\title{
Head-Up Tilt Rapidly Compromises Hemodynamics in Healthy Anesthetized Swine
}

\author{
M. MLCEK ${ }^{1}$, J. BELOHLAVEK ${ }^{2}$, M. HUPTYCH ${ }^{3}$, T. BOUCEK ${ }^{2}$, T. BELZA ${ }^{2}$, S. LACKO $^{1}$, \\ P. KRUPICKOVA ${ }^{2}$, M. HRACHOVINA ${ }^{3}$, M. POPKOVA ${ }^{1}$, P. NEUZIL $^{4}$, O. KITTNAR ${ }^{1}$ \\ ${ }^{1}$ Institute of Physiology, First Faculty of Medicine, Charles University in Prague, Czech Republic, \\ ${ }^{2}$ Second Department of Medicine, First Faculty of Medicine, Charles University in Prague, Czech \\ Republic, ${ }^{3}$ Department of Cybernetics, Faculty of Electrical Engineering, Czech Technical \\ University in Prague, Czech Republic, ${ }^{4}$ Department of Cardiology, Na Homolce Hospital, Prague, \\ Czech Republic
}

Received March 31, 2015

Accepted August 3, 2015

On-line December 15, 2015

\section{Summary}

The aims were to explore the effect of head-up tilt (HUT) to 30 and 60 degrees on hemodynamics and tissue oxygenation in anesthetized healthy swine. The data serve as a reference for a study of resuscitation efficacy at HUT such as during transport. Nine healthy swine $(49 \pm 4 \mathrm{~kg})$ were anesthetized and multiple sensors including myocardial pressure-volume loops catheter, carotid flow probe, blood pressure catheters, near infrared spectroscopy (NIRS) tissue oximetry and mixed venous oximetry (SVO2) catheter were introduced and parameters continuously recorded. Experimental protocol consisted of baseline in supine position (15 min), 30 degrees HUT (15 min), recovery at supine position (15 $\mathrm{min}$ ) and 60 degrees HUT (5 min). Vacuum mattress was used for body fixation during tilts. We found that 30 and 60 degrees inclination led to significant immediate reduction in hemodynamic and oximetry parameters. Mean arterial pressure $(\mathrm{mm} \mathrm{Hg})$ decreased from 98 at baseline to 53 and 39, respectively. Carotid blood flow dropped to $47 \%$ and $22 \%$ of baseline values, end diastolic volume to $49 \%$ and $53 \%$ and stroke volume to $47 \%$ and $45 \%$ of baseline. SVO2 and tissue oximetry decreased by 17 and 21 percentage points. The values are means. In conclusions, within minutes, both 30 and 60 degrees head-up tilting is poorly tolerated in anesthetized swine. Significant differences among individual animals exist.

\section{Key words}

Head-up tilt • Hemodynamics • Pressure-volume loop / Cardiac output $\bullet$ Swine $\bullet$ Oximetry

\section{Corresponding author}

M. Mlcek, Charles University in Prague, First Faculty of Medicine, Institute of Physiology, Albertov 5, 12800 Prague 2, Czech Republic. E-mail: mikulas.mlcek@staff.cuni.cz

\section{Introduction}

Maintaining adequate perfusion during cardiopulmonary resuscitation (CPR) effort remains a challenge. Furthermore, during CPR it is sometimes necessary to transport the patient in head-up tilted position such as inside the buildings (climbing the stairs or transport in space-restricted elevators). At present, it is not known how harmful such tilting is or conversely, whether it can be beneficial as recently suggested by Debaty et al. (2015).

Head-up tilting is a challenge to hemodynamics even in healthy subjects. Full power of several compensatory mechanisms needs to be engaged to maintain peripheral blood flow (Rowel 1993). These include vasoconstriction - both arterial and venous, increased myocardial contractility, baroreceptor reflex and its orthostatic adjustment, muscular and diaphragmatic pump (Miller et al. 2005) local myogenic reactions and more (Stewart 2012). Normal blood volume, normal oxygen carrying capacity and intact intrinsic vascular structure are other prerequisites of orthostatic tolerance.

Under the conditions of cardiac arrest, hypoxia, acidosis and subsequent CPR with multiple drugs 
administration including general anesthetics many of the abovementioned mechanism can be seriously compromised. Nevertheless, available knowledge regarding tilting during $\mathrm{CPR}$ is scarce. Recently, the interesting results were reported by Debaty et al. (2015). In a porcine CPR at 30 degrees HUT, an increased cerebral perfusion was observed despite a decrease in systemic arterial pressure and carotid blood flow by about half compared to horizontal position.

In the present study, the effect of head-up tilting to 30 and 60 degrees in healthy anesthetized swine fixed in vacuum mattress was explored in the setup similar to that of ongoing resuscitation. In order to differentiate between the effects of tilting and the effects that are secondary to cardiac arrest and subsequent CPR healthy animals with spontaneous rhythm were observed in this study. Swine is a commonly used biomodel in biomedical research including resuscitation studies. While there are similarities with human, the concern also exists that there could be differences in the response to orthostatic stress. Based on published data it was expected that 30 degrees tilt would be tolerated and 60 degrees would severely compromise hemodynamics.

\section{Methods}

\section{Preparation and anesthesia}

The study was approved by institutional care and use committee and was performed in accordance with European Guidelines on Laboratory Animal Care. Nine healthy swine $(48 \pm 4.2 \mathrm{~kg})$ were used. Following the premedication with midazolam $0.3 \mathrm{mg} / \mathrm{kg}$ and ketamine $20 \mathrm{mg} / \mathrm{kg}$ i.m. the marginal ear vein access was secured and animal transported to the lab. Here, initial propofol bolus (2 $\mathrm{mg} / \mathrm{kg})$ was administered and animal was orotracheally intubated and connected to mechanical ventilator. Tidal volume was set to $8 \mathrm{ml} / \mathrm{kg}$ and respiratory rate was adjusted to keep end-tidal $\mathrm{CO}_{2}$ (etCO2) between $38-42 \mathrm{~mm} \mathrm{Hg}$. Anesthesia was maintained with continuous i.v. propofol (6-8 mg/kg.h) midazolam (0.1-0.2 mg/kg.h) and morphin (0.1-0.2 mg/kg.h). Heparin bolus (100 IU/kg) was given initially followed by continuous administration of $50 \mathrm{IU} / \mathrm{kg} . \mathrm{h}$ so that activated clotting time (ACT) was maintained over $200 \mathrm{~s}$. Normal saline was administered at $500 \mathrm{ml} / \mathrm{h}$ until central venous pressure (CVP) was 5-6 mm Hg. Saline drip was then continued at $5 \mathrm{ml} / \mathrm{kg} . \mathrm{h}$ to maintain fluids balance. Multiple intravascular sheaths were inserted percutaneously for later placement of measurement catheters.

\section{Monitored parameters}

ECG, pulse oximetry (SPO2), rectal temperature and etCO2 were monitored noninvasively by patient monitor (LifeScope TR, Nihon Kohden, Japan). Regional tissue oximetry (rSO2) was monitored by NIRS oximetry (INVOS Oximeter, Covidien, USA) in forehead and right thigh. Pulmonary artery catheter (CCO V, Edwards Lifesciences, USA) was placed into pulmonary artery via femoral vein. It provided continuous cardiac output (CCO), mixed venous saturation (SVO2) and pulmonary artery pressure measurement. High fidelity pressurevolume catheter (type 7F VSL, Scisense-Transonic, USA) was inserted via the left carotid artery into the left ventricle under fluoroscopy control and pressure-volume data were obtained by admittance method (Raghavan et al. 2004). Blood pressure was measured directly via catheters placed in right femoral artery, carotid artery, pulmonary artery and superior vena cava by means of fluid-filled transducers (Truwave, Edwards Lifesciences, USA) placed at the heart level. Transient ultrasound flow probe (PSB, Transonic, USA) was implanted around right carotid artery to provide continuous volumetric flow measurement. Intracranial pressure (ICP) sensor (Neurovent-P, Raumedic, Germany) was inserted into parietal cortex via a burr hole $2 \mathrm{~cm}$ laterally from the midline. Measured parameters were sampled at $400 \mathrm{~Hz}$ by Powerlab A/D converter and continuously recorded to personal computer running Labchart Pro software (AD Instruments, USA).

\section{Positioning}

After all the systems were in place, animals were immobilized in resuscitation vacuum mattress that was shaped around the body and fixed to the motorized tilting bed. Additionally, the front legs were stretched above head and tied to the tilting bed. Care was taken that flushed pressure sensors were placed so that they would remain at the heart level during the whole protocol including tilting.

\section{The protocol}

The protocol is summarized in Figure 1. After minimum of 15 min stabilization the baseline values were recorded and the animals were tilted to 30 degrees (headup) for $15 \mathrm{~min}$. Then the position was resumed to horizontal and $15 \mathrm{~min}$ hemodynamic stabilization followed. Subsequent 60 degrees tilt lasted for $5 \mathrm{~min}$. The time to position was 40 and $60 \mathrm{~s}$, respectively. The tilt was interrupted prematurely in case of hemodynamic 
intolerance defined as a decline in mean arterial pressure below $40 \mathrm{~mm} \mathrm{Hg}$ or rSO2 below $40 \%$ or carotid flow below $50 \mathrm{ml} / \mathrm{min}$ for longer than $2 \mathrm{~min}$.

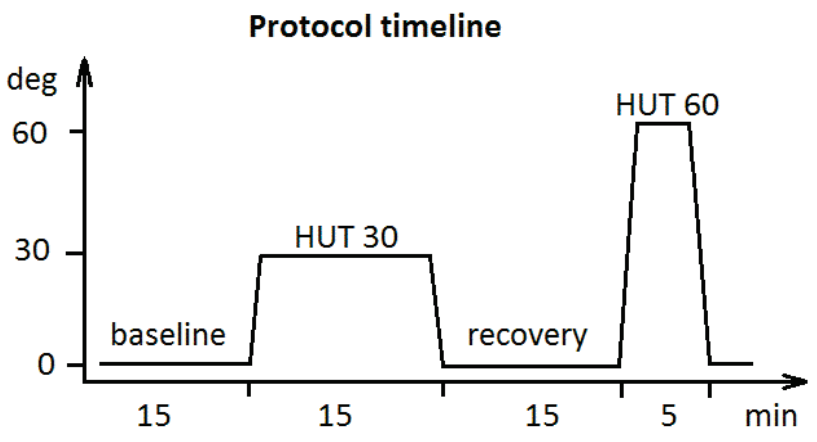

Fig. 1. Study protocol outline - Sequence of protocol phases. Horizontal axis represents sequence and duration of individual phases in minutes. HUT 30, HUT 60 - head-up tilt to 30 (60) degrees. Vertical axis represents degree of head-up inclination (deg).

\section{Statistics}

Shapiro-Wilk normality test was used to prove the normal distribution of analyzed parameters. Thus statistical analysis was performed by analysis of variance (ANOVA) for repeated measurements and paired t-test.

\section{Results}

Baseline

After anesthesia induction and stabilization, the baseline physiological parameters attained normal values that are summarized in Table 1. Significant interindividual variation was observed in carotid blood flow that ranged from 207 to $510 \mathrm{ml} / \mathrm{min}$. This variability correlated neither with body size nor with cardiac output.

\section{Head-up tilting}

The results are summarized in Table 1 and Figure 2. Tilting to 30 degrees rapidly compromised hemodynamics and tissue oxygenation. Arterial pressure, carotid blood flow, left venticular stroke volume and end diastolic volume fell to about $50 \%$ of respective baseline values. Intracranial pressure decreased by $16 \mathrm{~mm} \mathrm{Hg}$, however, after eliminating the effect of hydrostatic pressure drop secondary to sensor elevation, the ICP reduction was $4.5 \mathrm{~mm} \mathrm{Hg}$. Hydrostatic pressure was calculated as $\sin (30 \mathrm{deg}) \times 30 \mathrm{~cm}$ (distance between right atrium and ICP sensor), that is $15 \mathrm{~cm} \mathrm{H}_{2} \mathrm{O}$ or $11.5 \mathrm{~mm} \mathrm{Hg}$. Then, the calculated cerebral perfusion pressure (mean arterial pressure minus intracranial pressure) decreased from $79.3 \pm 12.8$ at horizontal position to $37.6 \pm 19.6$ at HUT 30. Central venous pressure changed from 6.8 to $1.8 \mathrm{~mm} \mathrm{Hg}$ suggesting reduced preload. At the same time, the ejection fraction remained unchanged. Tissue oxygenation and mixed venous oxygenation decreased to $46.5 \%$ and $31.5 \%$, respectively (i.e. $74 \%$ and $64 \%$ of their baseline values) demonstrating an increased $\mathrm{O} 2$ extraction partially compensating reduced oxygen delivery.

Table 1. Averaged hemodynamic and oximetry results for baseline (0 DEG), 30 and 60 degrees head-up tilt (HUT 30 and HUT 60).

\begin{tabular}{|c|c|c|c|c|c|c|c|c|c|}
\hline \multirow{2}{*}{$\begin{array}{l}\text { Parameter } \\
\text { (unit) }\end{array}$} & \multicolumn{2}{|c|}{ 0 DEG } & \multicolumn{2}{|c|}{ HUT 30} & \multicolumn{2}{|c|}{ HUT 60} & \multicolumn{2}{|c|}{ p values } & \multirow[t]{2}{*}{$\mathbf{n}$} \\
\hline & mean & SD & mean & SD & mean & SD & 0 vs. 30 & 30 vs. 60 & \\
\hline$M A P(m m ~ H g)$ & 98.22 & 13.52 & 52.67 & 18.81 & 38.88 & 9.28 & 0.001 & 0.015 & 9 \\
\hline$C A R(\mathrm{ml} / \mathrm{min})$ & 358.86 & 108.58 & 140.29 & 55.08 & 53.29 & 33.27 & 0.001 & 0.015 & 7 \\
\hline$S V(m l)$ & 88.88 & 22.36 & 41.75 & 14.59 & 40.00 & 16.53 & 0.001 & n.s. & 8 \\
\hline$E D V(m l)$ & 157.38 & 33.00 & 76.38 & 33.87 & 82.63 & 53.25 & 0.001 & n.s. & 8 \\
\hline$E F(\%)$ & 56.75 & 9.72 & 57.63 & 16.04 & 56.50 & 17.80 & n.s. & n.s. & 8 \\
\hline$C V P(m m ~ H g)$ & 6.89 & 1.54 & 1.83 & 2.00 & -5.63 & 4.84 & 0.001 & 0.001 & 9 \\
\hline$I C P(m m ~ H g)$ & 18.29 & 7.20 & 2.29 & 7.52 & -7.43 & 8.10 & 0.001 & 0.001 & 7 \\
\hline SVO2 (\%) & 49.71 & 7.06 & 31.57 & 12.27 & 26.57 & 10.03 & 0.001 & n.s. & 7 \\
\hline rSO2 (\%) & 64.00 & 11.10 & 46.52 & 7.82 & 44.00 & 7.18 & 0.013 & n.s. & 6 \\
\hline
\end{tabular}

MAP - mean arterial pressure, CAR - carotid blood flow, SV - left ventricular stroke volume, EDV - left ventricular end-diastolic volume, EF - left ventricular ejection fraction, CVP - central venous pressure, ICP - intracranial pressure, SVO2 - mixed venous blood saturation, rSO2 - regional oxygen saturation (forehead), n - number of samples 
Tilting to 60 degrees compared to 30 degrees resulted in dramatically reduced carotid blood flow $90 \pm 109 \mathrm{ml} / \mathrm{min}$ vs. $404 \pm 164 \mathrm{ml} / \mathrm{min}$ ( $22 \%$ of baseline), further reduced CVP $(-5.6 \mathrm{~mm} \mathrm{Hg})$ and mean arterial
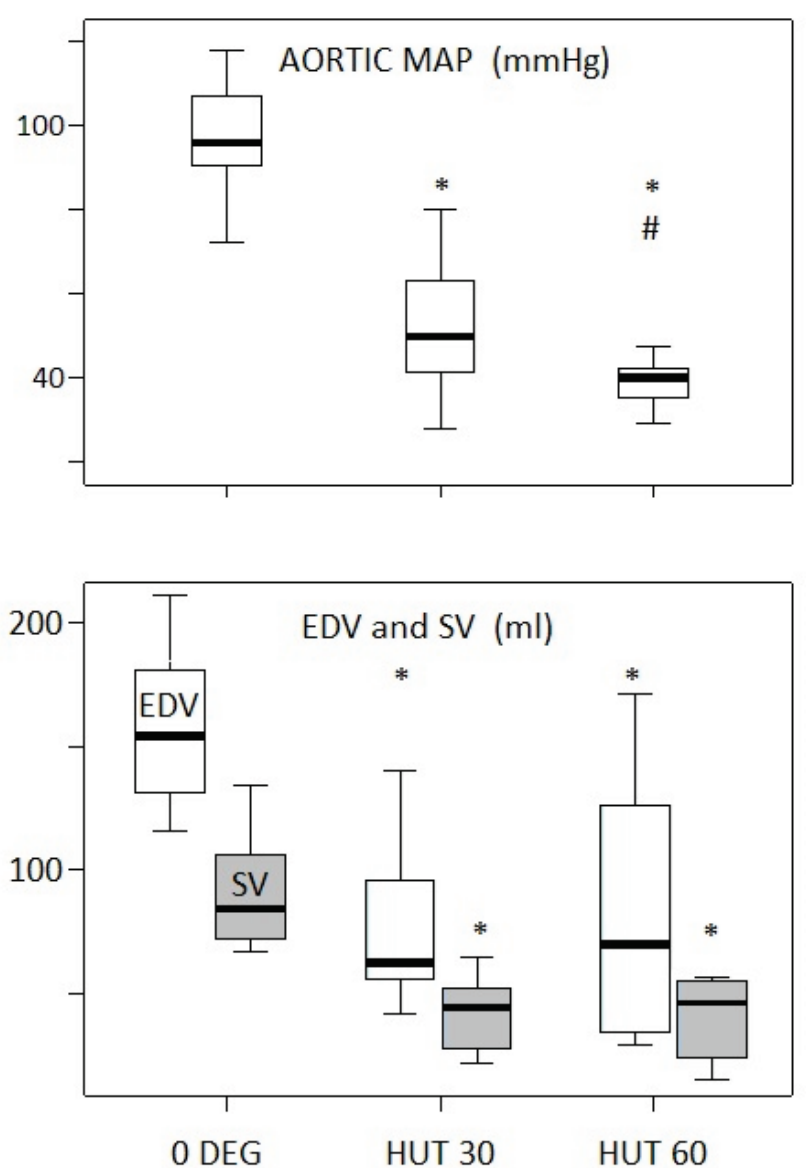

pressure $39 \mathrm{~mm} \mathrm{Hg}$ (40\% of resting values). However, other parameters (EDV, SV, SVO2, rSO2) did not differ significantly between 30 and 60 degrees tilt. Left ventricular ejection fraction again remained unchanged.
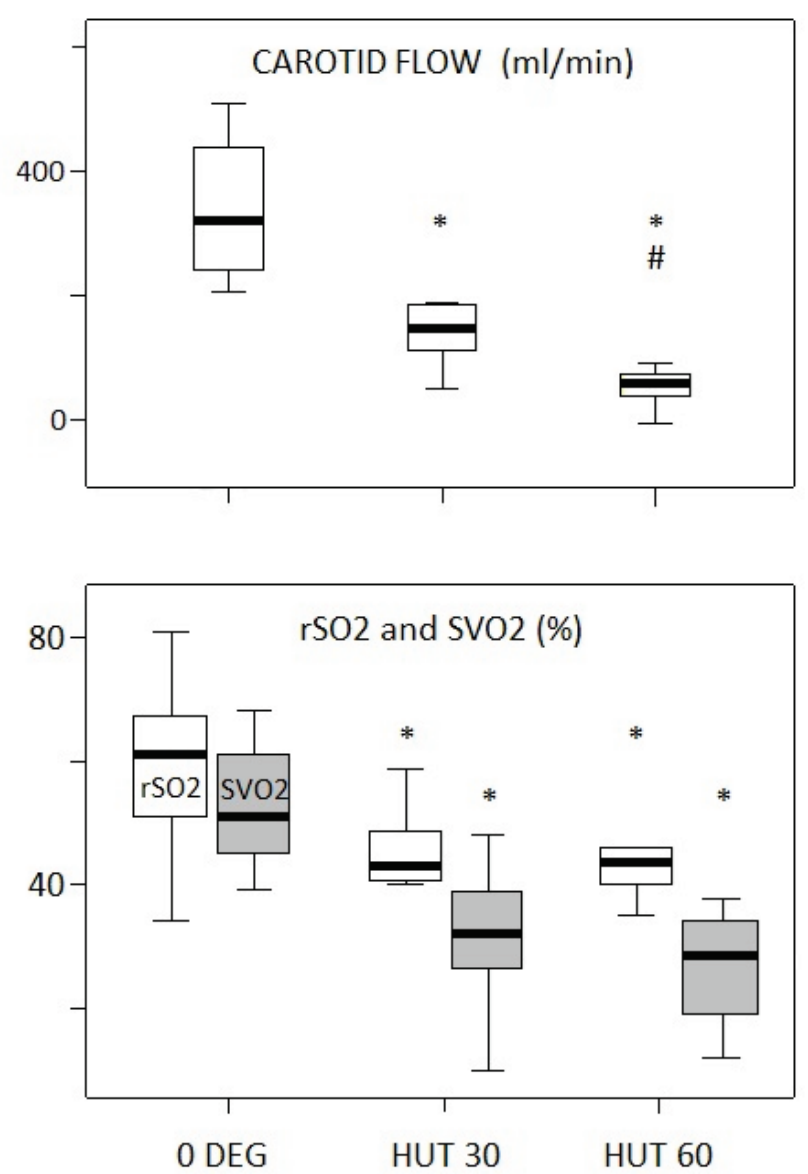

Fig. 2. Hemodynamic and oximetry parameters at baseline (0 DEG) 30 and 60 degrees head-up tilt. (HUT 30, HUT 60). MAP - mean arterial pressure, CAR - carotid blood flow, EDV - left ventricular end-diastolic volume (white), SV - left ventricular stroke volume (gray), rSO2 - regional oxygen saturation (white), SVO2 - mixed venous blood saturation (gray). $* \mathrm{p}<0.001$ vs. 0 deg. \# $\mathrm{p}<0.015$ vs. HUT 30. Box plots: inside the box there are $50 \%$ of values, thick line represents median. Top (bottom) $25 \%$ of values are above (below) the box as indicated by vertical lines.

A representative time course of hemodynamic parameters changes after both tilts is presented in Figure 3. The time to the position was $40 \mathrm{~s}$ and $60 \mathrm{~s}$ and already during this period the monitored parameters rapidly decreased near to the minimal values in each position. Then, in most animals, the hemodynamics stabilized throughout the rest of HUT period and little hemodynamic compensation was observed if any. Oximetry parameters declined less rapidly than hemodynamic ones. With the return to horizontal position, all the parameters recovered promptly excluding stroke volume that required more than $10 \mathrm{~min}$ to return to the baseline.

\section{Intolerance}

Two and four animals did not tolerate tilting to 30 and 60 degrees, respectively and thus the HUT maneuver was interrupted.

\section{Complications}

All the animals survived the study and there were no complications. However, tilting to 60 degrees represented a challenge to securing all the monitoring systems in place so that the data recording was reliable. Despite the effort to prevent sensors misplacement some signals had to be excluded from further processing for poor recording quality. 

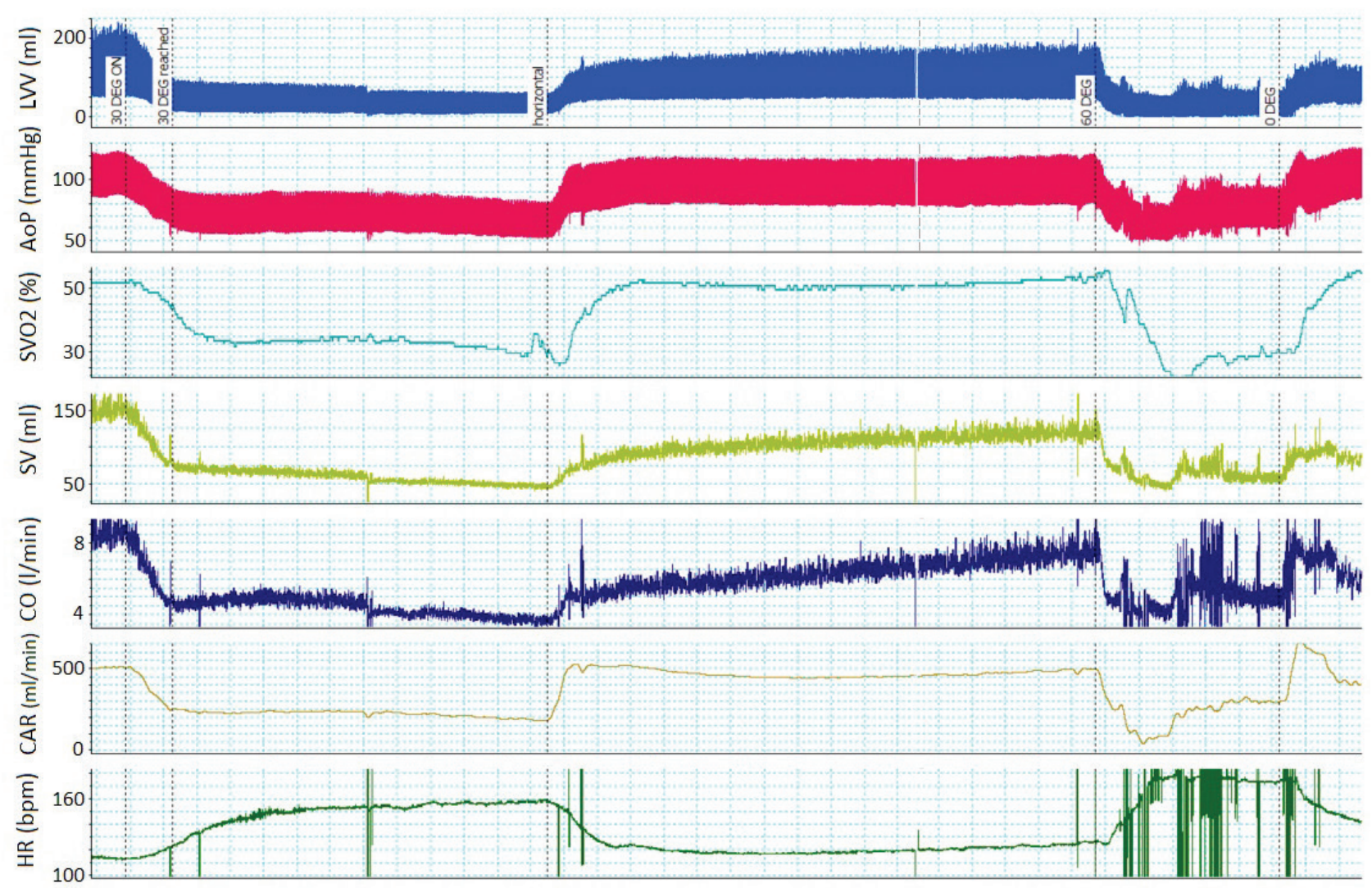

\begin{tabular}{|l|l|l|l|}
\hline O DEG & HUT 30 & recovery & HUT 60 \\
\hline
\end{tabular}

Fig. 3. Sample continuous data traces from one animal. Horizontal axis represents the protocol timeline. 0 DEG - baseline at horizontal position, HUT 30 and HUT $60-30$ and 60 degrees head-up tilt. LVV left ventricular volume, AoP - aortic blood pressure, SVO2 - mixed venous blood saturation, $\mathrm{CO}$ - cardiac output, CAR - carotid blood flow, HR - heart rate

\section{Discussion}

While it was expected that head-up tilting to 60 degrees will compromise and possibly collapse hemodynamics, it was surprising to find that already 30 degrees elevation has an immediate significant adverse effect that was even not tolerated by some animals. Hemodynamic destabilization in our setup was thus greater than in other studies exploring head-up tilting in pigs. In our study, the 30 degrees HUT in anesthetized swine resulted in about $50 \%$ decrease in mean arterial pressure, carotid blood flow, end diastolic volume and stroke volume immediately during tilting (see Fig. 3). Peripheral NIRS oximetry (rSO2) dropped to saturation values at around $40 \%$ which indicated tissue hypoperfusion while normal values are near $60 \%$. In a study by Klopfenstein a 20 degrees HUT resulted only in moderate decrease in cardiac output ( $-20 \%$ of baseline) and mean arterial pressure $(-10 \%$ of baseline $)$ (Klopfenstein et al. 1998). In 30 degrees tilt with capnoperitoneum, Junghans et al. (2006) reported similar changes in hemodynamics to Klopfenstein study: cardiac output was reduced by $24 \%$, mean arterial pressure by $6 \%$ and stroke volume by $30 \%$. Our results are more similar the resuscitation study reported by Debaty et al. (2015). In 30 degrees tilt during cardiac arrest treated by automated chest compressions they found considerable decrease in arterial pressure (roughly $50 \%$ ) and carotid flow (more than $50 \%$ ). Despite this they have seen improved brain tissue perfusion at 30 degrees HUT compared horizontal position, presumably due to improved venous return from brain circulation.

There could be several reasons for markedly compromised hemodynamics in our study compared to others.

General anesthesia. Propofol, an i.v. anesthetic used in the study, is known to inhibit hemodynamics by various effects (contractility, arterial vasoconstriction, venous tone). It has been suggested that in clinically relevant doses venous depression might be the most pronounced effect of propofol (Muzi et al. 1992). This would be in line with our results of reduced end-diastolic 
volume but preserved ejection fraction and increased arterial resistance. In other studies, inhalation anesthetics are commonly administered, but these would certainly not be used during CPR.

Venous return restriction. The animal fixation by means of vacuum mattress could interfere with the venous return. Though rather homogenous pressure is expected, it cannot be excluded that the amount of pressure the mattress exerts on the body and the location of this pressure could result in variable chest and abdominal compression. The effect could then range from beneficiary centralization of venous blood or an "autotransfusion" effect to adverse blood pooling in subdiaphragmatic region, specifically if combined with tilting. In laparoscopic patients it has been reported, that intra-abdominal pressure increase by $12 \mathrm{~mm} \mathrm{Hg}$ combined with 30 degrees HUT reduces cardiac output by $35 \%$ and stroke volume by $25 \%$ (Junghans et al. 2006). In present study, there were no signs of consistent changes in central venous pressure and/or airway pressure following the mattress inflation. However, no direct information was recorded concerning the pressure or flow in subdiaphragmatic region.

Animal species. It can be argued that swine posture is naturally horizontal resulting in poor tolerance to more vertical position; however, we consider this a less likely reason. The results of other studies demonstrate reasonable tolerance of anesthetized pigs to tilting. Also, the elevation to 30 degrees represents a position that in swine can physiologically occur during daily life activities.

Fluid depletion. Hypovolemia was unlikely in the current study since enough infusions were administered so that CVP was always above $6 \mathrm{~mm} \mathrm{Hg}$ prior to baseline measurement. However, in human medicine, some degree of hypovolemia can be rather common.

Several hypotheses/conclusions can be drawn from our observations. Even in the absence of cardiac arrest hence in the absence of severe metabolic imbalance, head-up tilting of anesthetized swine in vacuum mattress can result in a critical reduction of hemodynamic parameters. This seems to be a consequence of reduced preload since ventricular filling and central venous pressure were markedly reduced while vascular resistance increased. Despite hemodynamic changes, myocardial perfusion remained preserved or well compensated since ejection fraction changed neither at 30 nor at 60 degrees tilt. Regarding the brain, decreased cerebral perfusion pressure and regional tissue oxygenation in forehead would suggest limited cerebral perfusion. However, it is still possible that preserved autoregulation in cerebral bloodstream and improved venous return could keep cerebral microcirculation preserved.

Whether similar effects can be seen in humans during standard out-of-hospital CPR remains to be elucidated. In fully conscious healthy human volunteers, a rapid passive tilting to 30 degrees provokes almost $20 \%$ decrease in stroke volume that is explained by blood pooling in dependent body regions (Toska and Walloe 2002). Thus it is quite likely that under CPR conditions preload restriction will be a very important determinant of hemodynamics and any situation limiting venous return such as hypovolemia, blood pooling and impeded vasoreactivity should be addressed promptly.

\section{Limitations}

There are several limitations that deserve attention and further investigation. First, no robust markers of cerebral tissue perfusion adequacy such as electrophysiology were employed. Second, the need for global anesthesia in the doses relevant for miniinvasive procedures did introduce effects that may not apply to typical clinical scenarios. Particularly the use of propofol might have influenced the results, specifically via vascular reactivity. Another anesthetic such as midazolam might provide more relevant data. Additionally, despite a great care the tilting did interfere with the accuracy of data recording since some moves such those of internal organs within body cavities could not have been avoided. This might result in greater data variability than expected. Finally, significant interindividual variability was noticed with some animals not tolerating the tilt. This might confound some of the observations. The study was neither aimed nor powerful enough for any subgroup analysis.

\section{Conclusions}

Head-up position significantly compromises hemodynamics in healthy anesthetized swine restrained in vacuum mattress, probably due to preload limitation. Some of the findings might apply to human resuscitation pathophysiology.

\section{Conflict of Interest}

There is no conflict of interest. 


\section{Acknowledgements}

Authors thank to Tereza Vavrikova, Alena Ehrlichova and Karel Kypta for invaluable technical assistance. This work was supported by SVV266503/2013 and Prvouk P35/LF1.

\section{Abbreviations}

AoP - aortic blood pressure, CAR - carotid blood flow,
CPR - cardiopulmonary resuscitation, CVP - central venous pressure, EDV - end diastolic volume, EF ejection fraction, HR - heart rate, HUT - head-up tilt, ICP - intracranial pressure, LVV - left ventricular volume, MAP - mean arterial pressure, NIRS - near infrared spectroscopy, rSO2 - regional tissue saturation, $\mathrm{SVO} 2$ - mixed venous blood saturation, SV - stroke volume.

\section{References}

DEBATY G, SHIN SD, METZGER A, KIM T, RYU HH, REES J, MCKNITE S, MATSUURA T, LICK M, YANNOPOULOS D, LURIE K: Tilting for perfusion: head-up position during cardiopulmonary resuscitation improves brain flow in a porcine model of cardiac arrest. Resuscitation 87: 38-43, 2015.

JUNGHANS T, MODERSOHN D, DORNER F, NEUDECKER J, HAASE O, SCHWENK W: Systematic evaluation of different approaches for minimizing hemodynamic changes during pneumoperitoneum. Surg Endosc 20: 763-769, 2006.

KLOPFENSTEIN CE, MOREL DR, CLERGUE F, PASTOR CM: Effects of abdominal $\mathrm{CO}_{2}$ insufflation and changes of position on hepatic blood flow in anesthetized pigs. Am J Physiol 275: H900-H905, 1998.

MILLER JD, PEGELOW DF, JACQUES AJ, DEMPSEY JA: Skeletal muscle pump versus respiratory muscle pump: modulation of venous return from the locomotor limb in humans. J Physiol 563: 925-943, 2005.

MUZI M, BERENS RA, KAMPINE JP, EBERT TJ: Venodilation contributes to propofol-mediated hypotension in humans. Anesth Analg 74: 877-883, 1992.

RAGHAVAN K, WEI CL, KOTTAM A, ALTMAN DG, FERNANDEZ DJ, REYES M, VALVANO JW, FELDMAN MD, PEARCE JA: Design of instrumentation and data-acquisition system for complex admittance measurement. Biomed Sci Instrum 40: 453-457, 2004.

STEWART JM: Mechanisms of sympathetic regulation in orthostatic intolerance. J Appl Physiol 113: 1659-1668, 2012.

TOSKA K, WALLOE L: Dynamic time course of hemodynamic responses after passive head-up tilt and tilt back to supine position. J Appl Physiol 92: 1671-1676, 2002. 